\title{
MEDIDAS PARA AMPLIAÇÃO À SANIDADE NA PISCICULTURA - A IMPORTÂNCIA DA ALIMENTAÇÃO DE QUALIDADE
}

\author{
Measures for Sanitary Expansion in Fish Farming - The Importance of Quality Food
}

Claucia Aparecida Honorato ${ }^{1}$

DOI: 10.30612/re-ufgd.v6i11.8818

Recebido: 24/09/2019 Aceito: 5/04/2019

\begin{abstract}
Resumo: Objetivou-se por meio destes eventos relacionar aspectos relevantes a implantação de um canal de conversa e assessoramento de produtores de peixes da comunidade Itamaraty, visando proporcionar suporte técnico para a produção sustentável e economicamente viável de peixes. Assim foi realizado a coleta e caracterização das dietas utilizadas por eles nas diferentes fases do desenvolvimento dos peixes cultivados nesta comunidade. As dietas foram analisadas e posteriormente foi realizado um dia de campo no qual foi repassado estas informações. Foi desenvolvido concomitantemente um folder informativo contendo sobre manejo alimentar de pacu submetido a estresse térmico. $\mathrm{Na}$ ocasião do evento este foi distribuído aos produtores rurais. $\mathrm{O}$ evento proporcionou uma demonstração teórico/prática sobre como a qualidade da dieta pode promover respostas benéficas no sistema de produção de peixe, com participação de produtores rurais, assentados e discentes de vários cursos. O evento supriu as necessidades de conhecimento do público, além de oferecer conhecimento de manejo alimentar dos peixes no período crítico da criação.
\end{abstract}

Palavras-chaves: aquicultura, extensão rural, qualidade da dieta, sistema de produção de pescado.

Abstract: The objective of this meeting was to relate relevant aspects to the implementation of a channel of conversation and advice of fish producers of the Itamaraty community, aiming to provide technical support for the production sustainable and economically Viable fish. Thus, the collection and characterization of the diets used by them were carried out in the different phases of the development of the fish cultivated in this community. The diets were analyzed and subsequently a field day was carried out in which this information was passed. An informative folder containing the Pacu food management underwent thermal stress was developed concomitantly. At the time of the event this was distributed to the rural producers. The event provided a theoretical/practical demonstration on how the quality of the diet can promote beneficial responses in the fish production system, with the participation of rural producers, seated, quilombolas and students of various courses. The event met the needs of knowledge of the public, besides offering knowledge of food management of the fish in the critical period of creation.

Key words: Aquaculture, rural extension, diet quality, fish production system

1 Professora da Universidade Federal da Grande Dourados clauciahonorato@ufgd.edu.br 
Introdução

O manuseio de peixes vivos é um assunto relevante para o sucesso do empreendimento aquícola, pois as manipulações e mudanças do ambiente soam inevitáveis, causando uma serie de reações fisiológicas. Os peixes submetidos ao manuseio sofrem alterações de sua condição metabólica inicial que constitui nos desvios da homeostasia. A intensidade das alterações e o tempo para retornam as condições fisiológicas iniciais são indicadores bastante úteis da qualidade da manipulação dos peixes INOUE et al., (2005).

Um dos fatores que provocam estresse em peixes são as variações de temperatura da água. As chuvas repentinas e intensas, frequentemente observadas no verão brasileiro ESTEVES (1988) são importantes causas de choque térmico devido à temperatura da água da chuva ser inferior aos dos corpos d'agua. O crescimento está correlacionado a temperatura da água. Em regiões onde o inverno é rígido, a temperatura da água é inferior a $17^{\circ} \mathrm{C}$, o crescimento dos peixes é quase nulo, podendo ocorrer problemas de parasitose. As mudanças bruscas de temperatura (choque térmico) é um importante agente estressor para peixes neotropicais podendo causar desbalanços nas reações bioquímicas destes animais heterotermos (TANCK et al., 2000).

$\mathrm{O}$ aproveitamento alimentar dos peixes também está associado à temperatura da água de cultivo. A eficiência da digestão nos animais ectotérmicos é afetada pela temperatura da água através de, pelo menos, cinco processos: consumo, nível de secreção de sucos gástricos, atividade enzimática, motilidade do trato gastrointestinal e taxa de absorção intestina. Carneiro (1990) descreve que o aumento na temperatura, dentro dos limites de tolerância térmica, aumenta a taxa metabólica, intensificando a busca pelo alimento, o consumo e provavelmente a digestão e a assimilação dos nutrientes, proporcionando maior velocidade de crescimento e ganho de peso.

Pesquisas sobre a atividade de alimentação segundo Jobling et al. (1983), têm importância prática sob vários pontos de vista e podem ampliar os conhecimentos sobre o ritmo de alimentação dos peixes, contribuindo assim para uma melhor utilização do alimento, levando a uma produção mais eficiente.

A obtenção de um manejo alimentar adequado de uma espécie depende de um conjunto de fatores que influenciam a ingestão dos alimentos tais como: quantidade e qualidade do alimento, tamanho, textura, cor, propriedades organolépticas do alimento, temperatura da água, oxigênio dissolvido, horário de arraçoamento, frequência e ritmo de alimentação, sistema de criação, teor de proteína e energia da ração. Muitos estudos foram realizados sobre os mecanismos 
que regulam a ingestão de alimentos, tempo de transito gastrointestinal, identificação e detecção do alimento (MAYUMEOSHIRO et al., 2012).

Para realizar o manejo alimentar adequado de uma espécie, deve ser estudado os fatores que influenciam a ingestão dos alimentos. A temperatura da água é um fator importante no cultivo de peixes, pois influência diretamente a ingestão de alimentos, bem como a quantidade de alimento a ser fornecido, horário de arraçoamento, frequência e ritmo de alimentação, nos diferentes sistemas de criação.

De acordo com Schimittou (1993), os peixes reduzem o consumo ou mesmo cessam a alimentação com a variação da temperatura da água para além da faixa ideal. O autor informa que a temperatura ideal para produção da maioria das espécies de água quente situa-se entre 25 e $28^{\circ} \mathrm{C}$. Sobre o comportamento alimentar de espécie neotropical, o manejo alimentar nos meses de temperaturas mais baixas devem ser realizadas duas vezes ao dia: meio do dia e à tarde (FRASCASCORVO et al.,2001).

Os estudos sobre o manejo alimentar justificam-se, pelo fato de que o componente mais relevante nos custos de produção da piscicultura é a alimentação, responsável por 24,85\% a $36,40 \%$ do custo total de produção (SCORVO FILHO et al., 1998). Além disso, um arraçoamento adequado contribui para a manutenção da qualidade da água de cultivo e dos efluentes da piscicultura.

\section{Material e Métodos}

A ação de extensão, MANEJO ALIMENTAR DE PACU SUBMETIDO A ESTRESSE TÉRMICO, vem sendo desenvolvido na Faculdade de Ciências Agrárias da Universidade Federal da Grande Dourados desde o ano de 2017. Mas foi abordada a ação ocorrida no ano de 2017 e 2018, que apresentou carga horária total de 500 horas. Sendo de abrangência regional, onde o público alvo foram os produtores rurais, estudantes universitários, professores, técnicos agrícolas e a comunidade geral da Grande Dourados.

Vários estudos, debates, congressos foram realizados sobre os problemas da alimentação de peixe, visando aprimorar os métodos ou procedimentos até então adotados para aumentar a eficiência, saúde e bem-estar dos peixes no período de inverno. Anterior a ação de manejo alimentar de peixes foi realizado uma consulta junto a comunidade para coleta de uma amostra de ração a qual foi submetido a analise tecnológicas: absorção de água. flutuabilidade, densidade, lixiviação de proteína. A composição bromatológica foi realizada as análises de matéria 
seca, proteína bruta, extrato etéreo e cinzas.

Em novembro de 2017, juntamente com Liga Acadêmica de Aquicultura no assentamento Itamaraty foi realizado um dia de campo abordando temas atuais relacionados à produção de peixes com ênfase na saúde, no qual foi apresentado os dados de controle de qualidade tecnológica das dietas que estavam sendo utilizadas nesta comunidade.

Foi demonstrado o efeito da genética no crescimento e bem estar dos peixes no cultivo. A importância da qualidade de água nos parâmetros de desenvolvimento e o impacto da nutrição no sistema de produção. Acoplado ao dia de campo foi realizado um levantamento dos trabalhos que abordam o impacto da suplementação em dietas para serem utilizadas no pré-inverno que visem à adaptação do sistema imunológico dos peixes.

O evento foi divulgado por meio de contato verbal, cartazes e por distribuição de folder em locais estratégicos.

\section{Resultados e Discussão}

A nutrição para prevenir a mortalidade passa por algumas questões que devem ser apontadas: As principais causas das enfermidades, as medidas para prevenção de doenças e as identificações de doenças.

\section{As Principais Causas:}

- $\quad$ Alta concentração de animais no mesmo local

- $\quad$ Mistura de lotes e origens

- Condição do ambiente: alimentação acima da capacidade de suporte, temperatura e PH ideal, baixa oxigenação da água.

- Manejo inadequado, falta de medicação e não separar os animais doentes, falhas na aplicação do medicamento, equipamentos para medicação sujas ou danificadas, local em que o medicamento é armazenado esteja sujo ou com a presença de insetos, falta de limpeza ou limpeza inadequada.

\section{Prevenção de Doenças:}

- Nutrição que atenda as enzimas nutricionais. 
Manter o local em que as tilápias vivem protegidas com redes, evitando à entrada de animais.

- $\quad$ Utilize meios de desinfecção para veículos na entrada da piscicultura.

- $\quad$ Manter o controle de qualidade da água.

- $\quad$ Controle de pragas

- Vitamina C

\section{Identificando as Doenças}

- Movimentos sem coordenação e chocam-se nas laterais.

- $\quad$ Torso ou alguma parte do corpo inflamada.

- Mantem as suas barbatanas coladas ao corpo e pouca movimentação.

- $\quad$ Observa-se a falta de apetite.

- $\quad$ Notam-se machas de cor branca pelo corpo, causadas por bactérias ou fungos.

- $\quad$ Penugens brancas algodoadas em alguma zona do corpo.

\section{Utilização de suplementos em dietas para peixes}

A alimentação é de fundamental importância para o cultivo intensivo de peixes, é importante observar a qualidade do alimento e a frequência e quantidade que são oferecidas aos animais, um animal subnutrido ficara estressado prejudicando a homeostase do organismo (OBA et.al., 2009).

Dentre os suplementos que ajudam a estimular o sistema imunológico de peixes está a vitamina C URBINATI e CARNEIRO (2004), a falta de vitamina C na dieta provoca letargia, anorexia, perda das escamas, deformações ósseas, acúmulo de leucócitos nas brânquias, hemorragias na superfície corporal e de órgãos internos e aumento dos danos provocados pelo estresse de manejo (MORAES et al., 2003).

O cromo pode também ser uma substancia que atue na diminuição do estresse em peixes devido seu efeito no metabolismo da glicose, principalmente em animais submetidos ao estresse de densidade de estocagem (FUJIMOTO et al., 2005).

\section{Experiências obtidas}

Foram levados para o dia de campo os cartazes científicos assim como algumas dietas de peixes com a finalidade de demostrar e realizar um debate sobre a qualidade das dietas 
ofertadas para tilápia do Nilo. Na prática, foi possível abordar os pontos positivos e negativos envolvidas nas escolhas das dietas. Além disso, o contato dos produtores com as dietas com aditivos alimentares como a vitamina $\mathrm{C}$ despertou outras possibilidades de suplementação dos peixes.

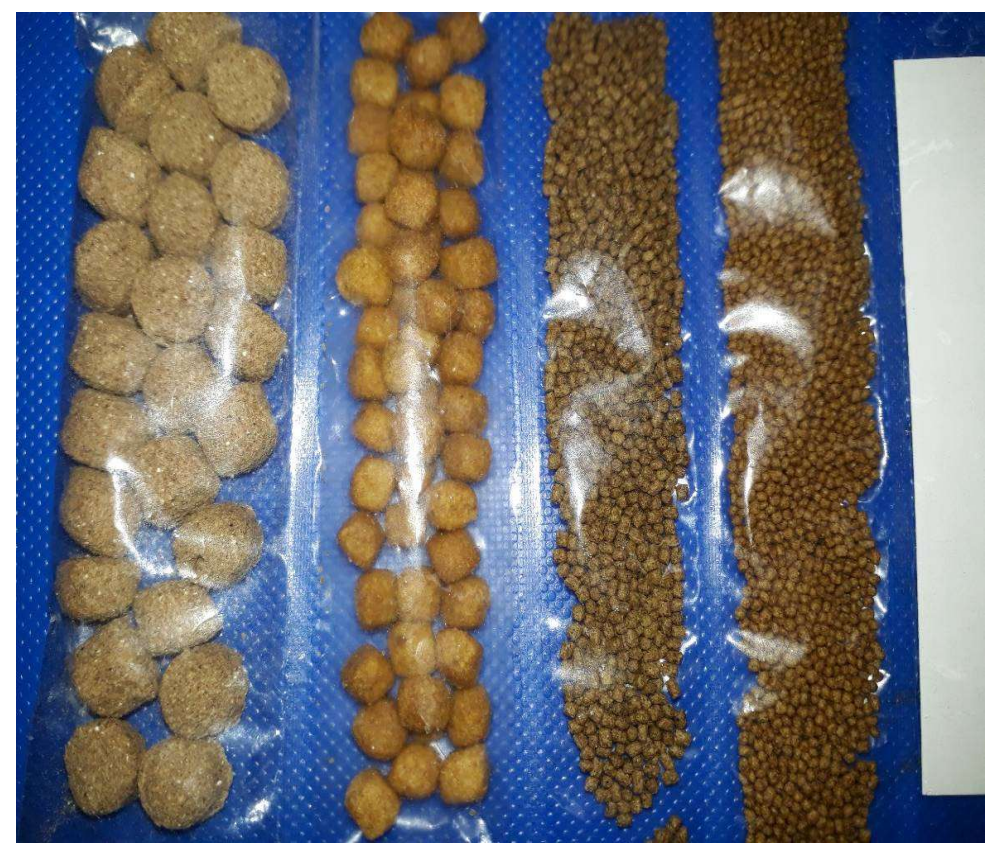

Figura 1 - rações de peixes para diferentes fases de cultivo

O trabalho gerou dados com as análises laboratoriais com o controle de qualidade (Tabela 1) de dietas. Com estes está sendo compilado um banco de dados para que possamos ter subsídios para tomada de decisão em relação a ração a ser ministrada ao sistema de cultivo.

Tabela 1 - Valores de qualidade tecnológica de dietas comerciais para tilápia do Nilo.

\begin{tabular}{|c|c|c|c|c|c|c|c|c|c|c|c|}
\hline \multirow[t]{2}{*}{ Dietas } & \multicolumn{2}{|c|}{$\begin{array}{c}\text { Peso do } \\
\text { pelet } \\
\text { (mg) }\end{array}$} & \multicolumn{2}{|c|}{$\begin{array}{c}\text { Tamanho } \\
\text { pelet } \\
(\mathrm{mm})\end{array}$} & \multicolumn{2}{|c|}{$\begin{array}{c}\text { Densidade } \\
\text { da dieta } \\
(\mathrm{g} / \mathrm{ml})\end{array}$} & \multicolumn{2}{|c|}{$\begin{array}{c}\text { Flu. } \\
\% \\
\end{array}$} & \multicolumn{2}{|c|}{$\begin{array}{c}\text { Absorção } \\
\text { de água } \\
\text { \% }\end{array}$} & $\begin{array}{l}\text { Lixivi_- } \\
\text { ação de } \\
\text { proteína } \\
(\mathrm{g} / \mathrm{dl})\end{array}$ \\
\hline & & & & & & 0,03 & 100,0 & & 366,0 & 57,8 & \\
\hline Final & 1,25 & 0,00 & 14,33 & 0,58 & 0,40 & $\begin{array}{r}8 \\
0,02\end{array}$ & 0 & 0,00 & $\begin{array}{c}0 \\
390,9\end{array}$ & $\begin{array}{c}5 \\
51,4\end{array}$ & 0 \\
\hline Cresc. & 0,15 & 0,01 & م م & 1,00 & 0,36 & $\begin{array}{r}6 \\
0,02\end{array}$ & 66,82 & 7,15 & $\begin{array}{c}1 \\
286,3\end{array}$ & 3 & 0 \\
\hline Cresc. II & 0,19 & 0,01 & 9,00 & 1,00 & 0,49 & $\begin{array}{r}5 \\
0,03\end{array}$ & 98,21 & 2,53 & $\begin{array}{c}6 \\
509,0\end{array}$ & $\begin{array}{l}6,43 \\
77,1\end{array}$ & 0,038 \\
\hline Juvenil I & 0,07 & 0,01 & 4,33 & 0,58 & 0,43 & $\begin{array}{r}8 \\
0,03\end{array}$ & 79,74 & 2,78 & $\begin{array}{c}9 \\
409,0\end{array}$ & $\begin{array}{c}4 \\
51,4\end{array}$ & 0,000 \\
\hline Juvenil II & 0,03 & 0,01 & 4,00 & 0,00 & 0,43 & $\begin{array}{r}6 \\
0,04\end{array}$ & 90,97 & 0,09 & $\begin{array}{c}9 \\
422,7\end{array}$ & $\begin{array}{c}3 \\
12,2\end{array}$ & 0,019 \\
\hline Alevino & 0,03 & 0,02 & 3,67 & 0,58 & 0,35 & 0 & 71,73 & 7,17 & 3 & 1 & 0,019 \\
\hline
\end{tabular}




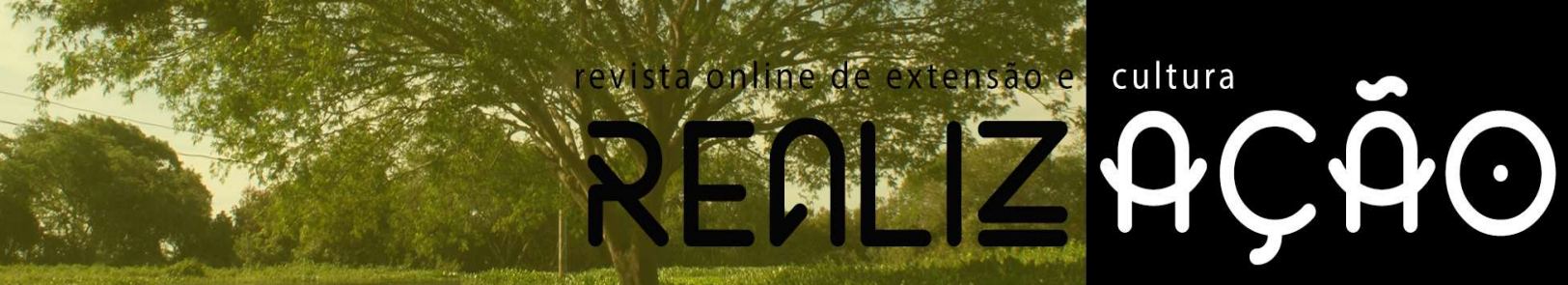

Cresc. $=$ Crescimento; Cresc. $=$ Crescimento II; Flu $=$ flutuabilidade

Atualmente há o questionado na piscicultura sobre o processamento aplicado para fabricação da ração, onde se tem dado bastante atenção às rações extrusadas para juvenis e adultos (BOOTH et al., 2000). Neste cenário as dietas extrusadas apresentam destaque pela praticidade de seu uso no cultivo. Utilizando-se o método de extrusão, têm-se obtido amidos modificados com maiores valores de solubilidade em água e com alta capacidade de absorver água, em virtude da gelatinização e dextrinização (GROSSMANN et al., 1988).

A partir dos resultados de pesquisa foi possível apresentar ao publico alvo as características das dietas que estão disponíveis no mercado para comercialização e se estas estão dentro do padrão de controle de qualidade.

Os dados de absorção de água e flutuabilidade apresentaram correlação positiva (Figura 2) o que reafirma que a gelatinização do amido é quem confere a característica de flutuar as dietas extrusadas.

Figura 3 - Valores relativos de flutuabilidade e de absorção de água das dietas.

Final Crescimento Juveni Alevino

campo é o que dá

suporte no

e renda, als

que à com

Além disso

à reflexão $\mathrm{t}$

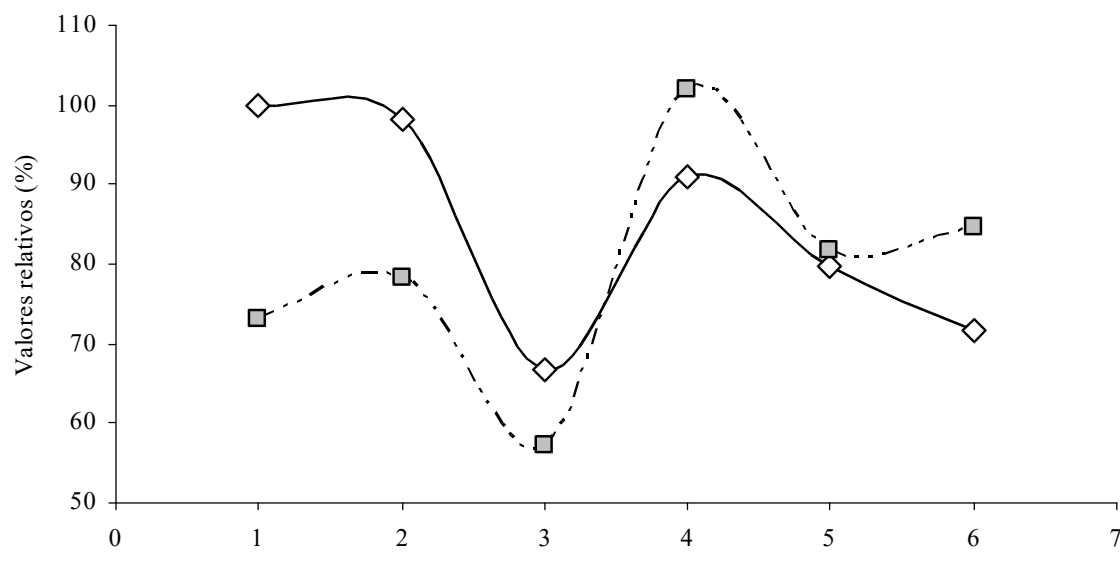

alimentos, emprego

niversitária faz com

sidade e Sociedade. ado que, submetido

$\smile$ Flutuabilidade

- ·- -.Absorção de água

entre o ensino e a

pesquisa, onde às analises implementadas serve para aulas práticas na graduação e pós-graduação e de pesquisa. Houve integração entre as áreas do conhecimento nos aspectos da interdisciplinaridade e multidisciplinaridade entre os discentes e docentes da Faculdade de Ciências Agrárias-FCA. A interdisciplinaridade depende fundamentalmente de uma atitude de colaboração dos pesquisadores frente ao desafio de uma prática coletiva, com o objetivo de se produzir conhecimento novo, unitário e crítico. No que tange a área de aplicação da área de nutrição de peixes conta com a 
colaboração de outras áreas de conhecimento como qualidade de água, sanidade de peixes.

A divulgação dos resultados permitiram que outras pessoas possam avaliar e, possivelmente, utilizar as informações que foram geradas e também poderá fazer com que a sociedade possa tomar conhecimento dos resultados de um trabalho de pesquisa e o que este representa para a coletividade. O trabalho promoveu a capacitação de recursos humanos para realização da atividade de dia de campo e verificou-se a capacitação dos agentes disseminadores de informações, é de fundamental importância para melhorar a qualidade e quantidade de pescado produzido.

O impacto social foi um reflexo das relações estabelecidas entre o público alvo e os elaboradores e condutores da ação de disseminação do conhecimento. A troca de experiência entre as questões técnico científica e a vivência dos produtores rurais enriqueceu a linha de trabalho e contribuindo diretamente para aumentar a produtividade e a renda das famílias participantes. A construção das ferramentas adequadas para um manejo racional que poderão disseminar estas informações para demais grupos de produtores fomentando a aquicultura nacional.

O impacto ambiental será um reflexo das boas ações de manejo alimentar que serão adotadas pelos produtores o qual selecionarão rações economicamente viáveis com melhor controle de qualidade para que haja menor lixiviação de proteínas diminuindo assim a emissão de compostos nitrogenados responsáveis pela eutrofização de ambientes aquáticos.

\section{Conclusão}

O manejo alimentar antes do período de inverno pode ser eficaz na diminuição dos custos de produção sem proporcionar prejuízos no desenvolvimento dos peixes. A possibilidade de redirecionar o manejo alimentar no cultivo de peixes e a adequação do regime alimentar podem ser uma alternativa de diminuir os custos de produção das empresas destinadas à produção de pescado. $\mathrm{O}$ aspecto social está vinculado ao aspecto ambiental uma vez que se pretende diminuir a excreção de compostos nitrogenados para meio ambiente.

O estudo de o manejo alimentar relacionado ao custo de arraçoamento a fím de aumentar a eficiência produtiva tem como cerne a nutrição de alevinos antes do inverno cultivados em tanque escavados. Cabe também destacar que o uso de dietas acrescidas de aditivos pode proporcionar consideráveis aumentos na produtividade. No entanto, não se tem protocolos de alimentação e para espécies nativas e não se sabe se há efetividade de resposta. A verificação da melhor eficiência produtiva dos peixes pela adoção de um protocolo alimentar eficiente preparando 
o peixe para época, refletirá em maior ganho para a cadeia de produção de pescado.

\section{Agradecimentos}

À Pró-Reitoria de Extensão e Cultura da Universidade Federal da Grande Dourados - UFGD. A comunidade Itamaraty pelo acolhimento da equipe técnica.

\section{Referências}

BOOTH, M.A., ALLAN, G.L.; WARNER-SMITH, R. Effects of grinding, steam conditioning and extrusion of a practical diet on digestibility and weight gain of silver perch, Bidyanus bidyanus. Aquaculture, v. 182, p. 287-299, 2000.

GROSSMANN, M.V.E.; EL- DASH, A.A.; CARVALHO, J.F. Extrusion cooking effects on hydration properties of moniac starsh. Arg. Biologyc. Tecnologyc., v. 31, p. 329-335, 1998.

GABRIEL, A.M.A.; SOUZA, R.; OLIVEIRA, E.R.; ROSSINI, L.C.; MONÇÃO, F.P.; RAMOS, M.B.M.; GIMENES, L.S.; PEREIRA, T.L.; SILVA, E.C.P. Orientação em apiários no Assentamento Amparo, Dourados-MS. Revista online de Extensão e Cultura Realização, v. 2, n.3, p. 36-41, 2015.

CARNEIRO, D. J. Efeito da temperatura na exigência de proteína e energia em dietas para alevinos de pacu, (Piaractus mesopotamicus) (HOLMBERG, 1887). São Carlos, UFSCAR, Tese de Doutorado, p. 55, 1990.

FRASCÁ-SCORVO, C. M. D.; CARNEIRO, D. J.; MALHEIROS, E. B. Comportamento alimentar do matrinxã Brycon Cephalus (GÜNTER, 1869) no período de temperaturas mais baixas. Bol. do Inst.de Pesca, vol. 27, n 1, p. 1-5, 2001.

FUJIMOTO, R. Y; CASTRO, M. P; MORAES, F. R. GONÇALVES, F. D. Efeito da suplementação alimentar com cromo trivalente em pacu, Piaractus mesopotamicus (Holmeberg, 1887) mantido em diferentes densidades de estocagem. Parâmetros Fisiológicos. Boletim Instituto da Pesca, v.31, n.2, p. 155-162.

INOUE, L. A. K. A.;AFONSO L. O. B.; IWAMA, G. K.; MORAES, G. Efeito do óleo de cravo na resposta de estresse do matrinxã (Brycon cephalus) submetido ao transporte. Acta Amazônica, Manaus, v.35, n. 2, p. 289-295, 2005.

JOBLING, M. Effect of feeding frequency on food intake and growth of Artic Charr, Salvelinud alpinus L. Journal Fish Biological, v. 23, p. 177-185. 1983.

MAYUMEOSHIRO, F.; FRAGA, T. L.; HONORATO, C. A. Tempo de transito gastrointestinal do pintado (Pseudoplatystoma sp.). Journal of Agronomic Sciences, Umuarama, v. 1, n. 2, p.128138, 2012.

MORAES, J. R. E; FREITAS, J. B; BOZZO, F. R; MORAES, F. R. E; MARTINS, M. L. A 
Suplementação alimentar com vitamina $\mathrm{C}$ acelera a evolução do processo Cicatricial em Piaractus mesopotamicus (HOLMBERG,1887). Boletim do Instituto de Pesca, v. 29, n.1 57- 67, 2003.

OBA, T. E; MARIANO, S. W; ROMAGUEIRA, L. Estresse em peixes cultivados: agravantes e atenuantes para o manejo rentável. In: TAVARES, D.M (Orgs), 2009. Embrapa, Amapá, Macapá.

SCHIMITTOU, H. P. High density fish culture in low volume cages. Singapore, American Soybean Association, p. 78, 1993.

SCORVO FILHO, J. D; MARTIN, N. B.; AYROZA, L. M. S. Piscicultura em São Paulo: custos e retornos de diferentes sistemas de produção na safra de 1996/1997. Informações Econômicas IEA, São Paulo, v. 28, n. 3, p. 41-60, 1998. 